\title{
Chronic Gastritis in Morbidly Obese Patients with Sleeve Gastrectomy
}

\author{
Ahmed Mohammad Makki ${ }^{1}$, Saleh Mohammad Aldaqal ${ }^{1}$, Shahad Hamadan Alorabi², Ismail Ahmed Nemri ${ }^{3}$,
} Maha Mohammad Alajami ${ }^{4}$

${ }^{1}$ Department of Surgery, Faculty of Medicine, King Abdulaziz University, Jeddah, Saudi Arabia

${ }^{2}$ Medical Student, Faculty of Medicine, King Abdulaziz Universty, Jeddah, Saudi Arabia

${ }^{3}$ House Officer, Faculty of Medicine, King Abdulaziz Universty, Jeddah, Saudi Arabia

${ }^{4}$ House Officer, Ibn Sina Faculty of Medicine, Jeddah, Saudi Arabia

Department of Surgery, King Abdulaziz University, Jeddah, Saudi Arabia

Type of article: Original

\begin{abstract}
Introduction: Obesity is a condition that has significant impact on public health. Recent exciting studies have linked chronic gastritis and H. pylori infection to obesity. Chronic gastritis has shown increased prevalence in obesity, more particularly H. pylori-related gastritis. This study aimed to determine the prevalence of chronic gastritis in morbidly obese patients who were subjected to sleeve gastrectomy, with focus on H. pylori-related gastritis. The aim was to demonstrate any influence on chronic gastritis of the surgical procedure or its postoperative course.

Methods: One hundred six morbidly obese patients were subjected to vertical sleeve gastrectomy in King Abdulaziz University Hospital (KAUH) from March 2014 to April 2015. After full pre-operative preparation, sleeve gastrectomy was carried out, followed by histological examination of the specimen to view the chronic gastritis and H. pylori-related cases. All epidemiological data of the patients, including age, gender, body mass index (BMI), as well as mean operative time, length of hospital stay, and post-operative complications were documented and analyzed by IBM-SPSS version 22 .

Results: Female patients presented the higher prevalence, i.e., $66 \%$ among the group of patients with sleeve resection, while $33 \%$ had chronic gastritis and $8.5 \%$ had $\mathrm{H}$. pylori-related gastritis.

Conclusion: This study highlights the high prevalence of chronic gastritis in morbidly obese patients with reference to the high incidence of $\mathrm{H}$. pylori-related gastritis among them, however no relationship was found between this infection and the post-operative outcome.

Keywords: morbid obesity, chronic gastritis, H. pylori, sleeve gastrectomy
\end{abstract}

\section{Introduction}

Obesity is an alarming public health problem and its prevalence is increasing worldwide (1). Gastritis is inflammation of the lining of the stomach due to several causes, including H. pylori infection, which is caused by gram negative bacteria that affect the gastric mucosa (2). Recent exciting studies have linked H. pylori infection to obesity (3). The prevalence of H. pylori in obesity and its possible impact on sleeve gastrectomy, which is used as therapeutic option for morbid obesity, require additional evaluation. The aim of this study was to show the prevalence of chronic gastritis in morbidly-obese patients who were subjected to sleeve gastrectomy, with focus on H. pylori-related gastritis to demonstrate any influence of chronic gastritis in the surgical procedure or its postoperative course.

\section{Corresponding author:}

Aldaqal S.M, Department of Surgery, King Abdulaziz University, Jeddah, Saudi Arabia.

E-mail: sdaqal@yahoo.com

Received: November 08, 2015, Accepted: December 23, 2015, Published: January 2016

iThenticate screening: December 10, 2015, English editing: December 28, 2015, Quality control: January 06, 2016

(C) 2015 The Authors. This is an open access article under the terms of the Creative Commons Attribution-NonCommercialNoDerivs License, which permits use and distribution in any medium, provided the original work is properly cited, the use is non-commercial and no modifications or adaptations are made. 


\section{Material and Methods}

This retrospective study was conducted for all sleeve gastrectomy procedures performed for morbid obesity in King Abdulaziz University Hospital (KAUH) in Jeddah, Saudi Arabia, from March 2014 to April 2015. Epidemiological data for each patient including age, gender, and BMI, were collected on a data sheet. Pre-operative preparation included a complete blood workup, abdominal ultrasound, and upper gastro-intestinal endoscopy. Vertical sleeve gastrectomy was performed by a skilled, well-trained, laparoscopic surgery team. Specimens were sent to histopathology to rule out any pathological finding and to diagnose gastritis and for H. pylori special stain. The mean operative time and the mean hospital stay were estimated for all cases. Particular attention was given to postoperative complications, with reference to their relationship with chronic gastritis. In-patient medications were only intra-venous fluids and Proton-pump inhibitor; the latter was prescribed as a home medication for two weeks after discharge from the hospital. Patients were instructed to come to the out-patient clinic for follow-up at intervals of one week, three months, six months, and one year. Regarding research ethics, approval for the study was obtained from the Hospital's Ethics Committee. The data were analyzed by IBM-SPSS version 22.

\section{Results}

This retrospective study included 106 morbidly obese patients who were admitted in KAUH through the out-patient clinic from March 2014 to April 2015. The ages ranged from 18 to 67 with a mean of 34. Seventy females and 36 males were involved in the study, i.e., 66 and $34 \%$ females and males, respectively. The mean BMI was 40.70 $\mathrm{kg} / \mathrm{m}^{2}$. The histopathology report showed 35 cases out $106(33.01 \%)$ had evident chronic gastritis, and nine of them were confirmed to have $\mathrm{H}$. pylori, accounting for $25.7 \%$ of chronic gastritis cases, $8.5 \%$ ) from all cases. The lamina propria of the gastric mucosa diagnosed with chronic gastritis showed increased plasma cells with lymphocytes and a few lymphoid follicles. The presence of neutrophils in the glandular or surface epithelial layer was a sign of activity. Helicobacter pylori bacteria, if present, were accentuated by Cresyl fast violet (CFV) stain. The mucosa harboring H. pylori contained lymphoid follicles with germinal centers in their lamina propria. The age of those cases with chronic gastritis, including the cases of $\mathrm{H}$. pylori-related gastritis, ranged from 21 to 55, and the mean age was 38. The nine cases that showed H. pylori were six females and three males. The mean BMI of gastritis cases was $40.75 \mathrm{~kg} / \mathrm{m}^{2}$, and the mean BMI for the whole group who had sleeve gastrectomy was almost the same of the BMI of the patients who had chronic gastritis with a $p$-value of 0.55 . The mean operative time was one hour and 20 minutes for all cases, including the gastritis cases, and the mean hospital stay was 2-3 days for all of the patients. Five cases developed post-operative complications (three with leaks, two with strictures), but none of them was found to have gastritis. The gender distribution of chronic gastritis and $\mathrm{H}$. Pylori are presented in Tables 1, 2.

Table 1. Gender distribution of chronic gastritis in all cases

\begin{tabular}{|l|l|l|l|}
\hline Patients & Female & Male & Total \\
\hline Chronic Gastritis Cases & 24 & 11 & $35(33 \%)$ \\
\hline Non-Gastritis Cases & 46 & 25 & $71(67 \%)$ \\
\hline Total & $70(66 \%)$ & $36(34 \%)$ & $106(100 \%)$ \\
\hline
\end{tabular}

Table 2. Gender distribution of H. pylori in Chronic Gastritis Cases

\begin{tabular}{|l|l|l|l|}
\hline Patients & Female & Male & Total \\
\hline Positive H. pylori cases & 6 & 3 & $9 / 35(25.7 \%)$ \\
\hline Negative H. pylori cases & 18 & 8 & $26(74.3)$ \\
\hline Total Number of Gastritis Cases & 24 & 11 & $35(100 \%)$ \\
\hline
\end{tabular}

\section{Discussion}

Obesity has become a major concern among physicians due to its large influence in metabolic, gastrointestinal, and hepatic diseases and due to its being an epidemic disorder in some countries (4). In the study in (4), the female-tomale ratio was 1.5: 1 , and this ratio suggests that women in the U.A.E have a higher prevalence of obesity than men (5). Our study showed the female-to-male ratio to be $2: 1$, further indicating a greater prevalence among females. The pathophysiology of obesity is affected by environmental and host agents, making the pathophysiology of obesity multifactorial (1). The various factors involved in the process include diet, in the lack of physical activity, the use of drugs, and genetic and neurophysiological factors. The development of obesity is affected by two important gastrointestinal hormones, i.e., leptin and ghrelin, which are involved in the dysregulation of the mechanisms that control food intake. Leptin suppresses the desire to intake food, thereby inducing weight loss. Ghrelin is secreted by the gastric mucosa and is thought to regulate eating behavior and weight balance. This peptide abruptly peaks just 
before a meal, and it decreases rapidly afterwards. It also has orexigenic effects and inhibits the secretion of leptin (6).

Gastritis refers to the irritation and/or inflammation of the lining of the stomach. This can occur gradually (chronic gastritis) or it may happen abruptly (acute gastritis). Yamoto et al. indicated that several studies of morbid obese patients have indicated that obesity is related to the high incidence of endoscopic and histologic gastritis. Thirtythree percent of our patients (35 out of 106) were proved to have chronic gastritis. Warren and Marshall in 1982 (7) first described Helicobacter pylori as gram negative, spiral-shaped bacteria. They stated that it's the main route of infection was feco-oral (8), and it is considered to be one of the main causes of gastritis. The prevalence of infection is higher in developing countries $(>80 \%)$ than it is in developed countries $(20-40 \%)(8)$. There is still considerable debate concerning the prevalence of $\mathrm{H}$. pylori infection in morbidly obese patients. The patients who were scheduled for bariatric surgery had a pre-operative prevalence of $\mathrm{H}$. pylori that ranged from $8.7 \%$ to $85.5 \%(9,10)$. $\mathrm{H}$. pylori was identified in 5.2\% of all cases and in 33.3\% of cases of gastritis in Raees et al.'s study (11), while, in our study, it was illustrated that H. pylori was found in 9/106 (8.5\%) of all cases, 9/35 (25.71\%) of gastritis cases, representing very similar results. There are two main reasons for the high concern about $\mathrm{H}$. pylori infection in obesity, i.e., (1) its possible relationship with BMI and (2) its acting as a negative factor in limiting access to bariatric surgery. The effects of $\mathrm{H}$. pylori on the production of gastric ghrelin still are controversial. In obese patients, H. pylori diminishes the density of gastric ghrelin-positive cells, which are associated with neutrophil activity, chronic inflammation, and glandular atrophy. The involvement of H. pylori in the pathophysiology of obesity is still unclear. Some authors, such as Osawa, have claimed that the H. pylori infection protects against obesity by decreasing secretion of ghrelin (12). However, other recent studies have failed to identify a relationship between H. pylori infection and reduced BMI (Caraboti et al. 2014). Erim et al. indicated that obesity can alter innate and adaptive immunity and that the immunological impairment was related to the grade of obesity, thereby producing less maturation of monocytes into macrophages and reducing the polymorphonuclear bactericidal capacity, which promote the development of gastritis (13). That there is a protective role of infection towards obesity seems apparent due to the significant increase in BMI after the successful elimination of H. pylori. However, the increased incidence and severity of infection observed in obese patients, due to their decreased immunity seem to counter the argument that $\mathrm{H}$. pylori infection has a protective role (14).

The probable explanation of these inconsistent results could be the pattern of H. pylori-related gastritis. Gastric extension, which generally starts with enteritis and progresses to pan-gastritis, alters the effect of H. pylori infection. Also, the occurrence of atrophy reduces the secretory activity, which subsequently decreases the production of ghrelin (15). Serology, which is used to diagnose H. pylori infection in most of studies, has a low diagnostic accuracy, but it is useful for excluding H. pylori infection. Positive serology should be confirmed by a test for active infection, such as the stool antigen assay, which has a sensitivity of $94 \%$ and a specificity of $92 \%$. Also, the urea breath test (UBT) can be used, and it has a sensitivity of 88\%-95\% and specificity of 95\%-100\%. Histological examination has excellent sensitivity and specificity, and it was used successfully in our study to diagnose H. pylori infection. According to Raees et al., candidates for the surgery should undergo a rigorous pre-operative evaluation, including double contrast upper gastrointestinal radiographic series. Our preoperative protocol followed the European guidelines, which specify upper gastrointestinal endoscopy before bariatric surgery to treat any lesions, including $\mathrm{H}$. pylori infection. This is due to the fact that such lesions may cause post-operative complications (16). However, patients schedule to undergo sleeve gastrectomy are presumed to have no significant gastric pathology. Even so, eradication should decrease the risk of gastro-duodenal peptic lesions in the gastro-jejunostomy site after gastric bypass (17). Laparoscopic sleeve gastrectomy has been advocated as a multipurpose restrictive procedure for obese patients (18) with low incidence of complications (19). The existing infection might represent a confounding and limiting factor in bariatric surgery due to the high incidence of post-operative symptoms and/or lesions in noneradicated patients. Our study did not show any relationship between chronic gastritis and the post-operative outcome, since Laparoscopic sleeve gastrectomy entails gastric resection with pylorus preservation. In theory, this antral resection removes the habitat of $\mathrm{H}$. pylori bacteria, thereby eradicating them without medical therapy, which is a cost-effective approach $(20,21)$.

\section{Conclusions}

Obesity, a condition that is occurring with high incidence worldwide, has significant adverse impacts on public health. Chronic gastritis showed increased prevalence in obesity, more particularly H. pylori-related gastritis. It was clearly confirmed in our study that $33.01 \%$ of the patients who had sleeve gastrectomy also had chronic gastritis 
with a high incidence $(8.5 \%)$ of $\mathrm{H}$. pylori-related gastritis, which indicated that obesity is a risk factor for chronic gastritis and H. pylori infection. Pre-operative investigations and eradication of infection are necessary, because the stomach is presumed to be free of pathogens even if the outcome is not affected. Further studies are necessary to obtain clinical results at long-term follow-up.

\section{Acknowledgments:}

The authors thank the King Abdulaziz University Hospital (KAUH) for its help in this study.

\section{Conflict of Interest:}

There is no conflict of interest to be declared.

\section{Authors' contributions:}

All authors contributed to this project and article equally. All authors read and approved the final manuscript.

\section{References}

1) Maksud FA, Alves JS, Diniz MT, Barbosa AJ. Density of ghrelin-producing cells is higher in the gastric mucosa of morbidly obese patients. Eur J Endocrinol. 2011; 165(1): 57-62. doi: 10.1530/EJE-11-0201, PMID: 21558140.

2) Onzi TR, d'Acampora AJ, de Araújo FM, Baratieri R, Kremer G, Lyra HF Jr, et al. Gastric histopathology in laparoscopic sleeve gastrectomy: pre- and post-operative comparison. Obes Surg. 2014; 24(3): 371-6. doi: 10.1007/s11695-013-1107-8, PMID: 24158738.

3) Liew PL, Lee WJ, Lee YC, Chen WY. Gastric ghrelin expression associated with Helicobacter pylori infection and chronic gastritis in obese patients. Obes Surg. 2006; 16(5): 612-9. doi: 10.1381/096089206776945002, PMID: 16687031.

4) Yamamoto S, Watabe K, Takehara T. Is obesity a new risk factor for gastritis? Digestion. 2012; 85(2): 10810. doi: 10.1159/000334680, PMID: 22269289.

5) Hajat C, Harrison O, Al Siksek Z. Weqaya: a population-wide cardiovascular screening program in Abu Dhabi, United Arab Emirates. Am J Public Health. 2012; 102: 909-14. doi: 10.2105/AJPH.2011.300290, PMID: 21940918, PMCID: PMC3483896.

6) Carabotti M, D'Ercole C, Iossa A, Corazziari E, Silecchia G, Severi C. Helicobacter pylori infection in obesity and its clinical outcome after bariatric surgery. World J Gastroenterol. 2014; 20(3): 647-53. doi: 10.3748/wjg.v20.i3.647, PMID: 24574738, PMCID: PMC3921474.

7) Brown LM. Helicbacter pylori: epidemiology and routes of transmission. Epidemiol Rev. 2000; 22: 283 -97. doi: 10.1093/oxfordjournals.epirev.a018040, PMID: 11218379.

8) Guisset M, Conton T, Rey P, Debonne JM. Helicobacter pylori infection in developing countries. Med Trop. 1997; 57: 77-82, PMID: 9289617.

9) Küper MA, Kratt T, Kramer KM, Zdichavsky M, Schneider JH, Glatzle J, et al. Effort, safety, and findings of routine preoperative endoscopic evaluation of morbidly obese patients undergoing bariatric surgery. Surg Endosc. 2010; 24: 1996-2001. doi: 10.1007/s00464-010-0893-5, PMID: 20135170.

10) Al-Akwaa AM. Prevalence of Helicobacter pylori infection in a group of morbidly obese Saudi patients undergoing bariatric surgery: a preliminary report. Saudi J Gastroenterol. 2010; 16: 264-7. doi: 10.4103/1319-3767.70610, PMID: 20871190, PMCID: PMC2995094.

11) Raess PH, Baird-Howell M, Aggarwal R, Williams N, Furth E. Vertical sleeve gastrectomy specimens have a high prevalence of unexpected histopathologic findings requiring additional clinical management. Surg Obes Relat Dis2015; 11(5): 1020. doi: 10.1016/j.soard.2015.01.002.

12) Osawa H. Ghrelin and Helicobacter pylori infection. World J Gastroenterol. 2008; 14: 6327-33. doi: 10.3748/wjg.14.6327, PMID: 19009647, PMCID: PMC2766113.

13) Erim T, Cruz-Correa MR, Szomstein S, Velis E, Rosenthal R. Prevalence of Helicobacter pylori seropositivity among patients undergoing bariatric surgery: a preliminary study. World J Surg. 2008; 32: 2021-5. doi: 10.1007/s00268-008-9608-7, PMID: 18581170.

14) Arslan E, Atilgan H, Yavaşoğlu I. The prevalence of Helicobacter pylori in obese subjects. Eur J Intern Med. 2009; 20: 695-7. doi: 10.1016/j.ejim.2009.07.013, PMID: 19818289.

15) El-Omar EM, Oien K, El-Nujumi A, Gillen D, Wirz A, Dahill S, et all. Helicobacter pylori infection and chronic gastric acid hyposecretion. Gastroenterology. 1997; 113: 15-24. doi: 10.1016/S00165085(97)70075-1, PMID: 9207257. 
16) Sauerland S, Angrisani L, Belachew M, Chevallier JM, Favretti F, Finer N, et al. Obesity surgery: evidence-based guidelines of the European Association for Endoscopic Surgery (EAES). Surg Endosc. 2005; 19: 200-21. doi: 10.1007/s00464-004-9194-1, PMID: 15580436.

17) Scheffel O, Daskalakis M, Weiner RA. Two important criteria for reducing the risk of postoperative ulcers at the gastrojejunostomy site after gastric bypass: patient compliance and type of gastric bypass. Obes Facts. 2011; 4 Suppl 1: 39-41. doi: 10.1159/000327340, PMID: 22027289.

18) Braghetto I, Korn O, Valladares H, Gutiérrez L, Csendes A, Debandi A, et al. Laparoscopic Sleeve Gastrectomy: Surgical Technique, Indications and Clinical Results. Obes Surg. 2007; 17(11): 1442-50. doi: 10.1007/s1 1695-008-9421-2, PMID: 18219770.

19) Dey A, Mittal T, Malik VK. Initial Experience with Laparoscopic Sleeve Gastrectomy by a Novice Bariatric Team in an Established Bariatric Center-A Review of Literature and Initial Results. Obes Surg. 2013; 23(4): 541-7. doi: 10.1007/s11695-012-0797-7, PMID: 23099854.

20) Ng SW, Zaghloul S, Ali H, Harrison G, Yeatts K, El Sadiq M, et al. Nutrition transition in the United Arab Emirates. Eur J Clin Nutr. 2011; 65: 1328-37. doi: 10.1038/ejcn.2011.135, PMID: 21772317, PMCID: PMC3304306.

21) Gagner M, Deitel M, Kalberer TL, Erickson AL, Crosby RD. The Second International Consensus Summit for Sleeve Gastrectomy. Surg Obes Relat Dis. 2009; 5(4): 476-85. doi: 10.1016/j.soard.2009.06.001, PMID: 19632647. 